\title{
The Design of Intelligent Home Alarming System Based on Wireless Sensor Network Technology
}

\author{
Xin Chen \& Weizhi Huang \\ School of Information and Communication Engineering \\ Tianjin Polytechnic University \\ Tianjin 300160, China \\ E-mail:chenhong_101@126.com
}

\begin{abstract}
Aiming at the characters of wireless sensor network and intelligent home alarming system, this article studied a sort of intelligent home alarming system based on wireless sensor network. Through establishing the structure frame of alarming system, in this article, we designed the sensor nodes of alarming system, put forward the hardware design methods of sensor nodes for the alarming system, designed the software of alarming system through the combination of software and hardware, adopted the license technology to enhance the security and veracity of the transmission of alarming information. The experiment indicated that the system had certain practical value because of its convenient operation and reliable stability.
\end{abstract}

Keywords: Wireless sensor network, Intelligent home, Alarming system, Nodes, CC2420

\section{Introduction}

Wireless sensor network centralizes the sensor technology, wireless communication technology and embedded computation technology, and it can perceive, supervise and collect information of perceptive object under various environments, and obtain exact information of perceptive object through the treatment of information. The wireless sensor network expands human ability to acquire information and connects physical information of objective world with transfer network, and it is applied not only in traditional network, but more in the occasions that the wire connection is unserviceable and the environment that people can not arrive. It has very extensive application foreground in many domains such as scientific research, environment supervision, intelligent home, medical supervision and control, traffic transfer, supervision of special goods, weapon equipment, agricultural breeding and industrial control (Huang, 2006, P. 621-624). With continual enhancement of human living level and the gradual increase of safety and prevention consciousness, people begin to put forward higher requirements for the home intelligence and security. As one important composing part of the intelligent home system, the performance of the alarming system directly influences the whole intelligent home system. To apply the wireless sensor network into the intelligent home alarming system and collect the environmental information of home through various sensors and transmit information to the home control center by the wireless mode could compensate the deficiency of wire equipment, and the wireless sensor network has many advantages such as cheap price, high reliability and easy emendation.

According to the characters of wireless sensor network and intelligent home, in this article, we put forward the project of the intelligent home alarming system based on wireless sensor network, and adopt the design of modularization to make it possess better transplanted property and expansibility.

\section{Basic structure of intelligent home alarming system}

Wireless sensor network (Egan, 2005, P. 14-19, Edgar, 2003, Zheng, 2005, P. 86-88, Wang, 2005, P. 59-62) is the network compose by many sensor nodes through the self-organization of wireless communication technology, and in the wireless sensor network system, every sensor node possesses the function of wireless communication, and the sensor unit of various measure points measures the parameter at the position and composes a wireless network and transmit the measured data to the monitor center by the wireless mode. The intelligent home alarming system based on wireless sensor network forms a wireless sensor network system composed by many sensor nodes including door magnetic switch, infrared sensor, fog sensor, gas leakage sensor and glass breaking alarm. In the alarming network, we adopt the star LAN structure which takes the wireless communication module connected with computer as the center node of the 
network, and communicates with any one sensor node in the network, and the sensor node could measure and sample various parameters in the home environment and transmit the collected data to the center node, and analyze and treat the data and orders from center node, then complete corresponding operation. The basic structure of the intelligent home alarming system is seen in Figure 1.

The alarming system adopt star LAN structure which could better extend the combination, easily increase the node of sensor, fulfill the uncertainty of sensor node distribution in the home, avoid wireless interference and uncertainty of alarming area because the sensor could be placed in room and isolation places, and comprehensively treat the data transmitted by various sensor nodes to analyze whether to send out alarming signals. The system could reduce the misinformation of alarming system to some extent and enhance the reliability of wireless alarming system. In the alarming system network, the main function of sensor node is to collect data, and transmit the data to the center node of the network by the wireless mode, then the center node comprehensively disposals the data and decides whether to give an alarm.

\section{Node design of sensor}

The node of sensor is the basic unit and the basic platform to compose the intelligent home alarming system. In the design process of sensor node, we adopt following design principles. We adopt common apparatus to select the components and consider the compatibility and currency at the same time. And we analyze the structure of node and adopt the design of modularization to divide the function of various modules. The nodes of sensor is composed by four parts including data collection module, data processing module, wireless communication module and power supply module (Sun, 2005, P.245), and it structure is seen in Figure 2.

\subsection{Module of data collection}

The module of data collection is composed by a group of sensors and corresponding A/D converters, and it is in charge of the information collection of supervision area and the corresponding data conversion.

\subsection{Module of data processing}

The module of data processing is in charge of the control of all sensor nodes, and it is the core component of the wireless alarming sensor node. The sensor node needs to use a processor with low power and strong function, and it could implement data exchange with wireless communication module, realize the collection and disposal of data, save energy for the power supply, and manage the operation status and inspection of the whole sensor nodes. In the design of sensor node, the selection of processor is very important. The selection of the processor with low power mainly considers the work pressure of SCM, the low power mode and the decrease of exterior circuits. In this article, we select the SCM of MSP430 series made by TI Company (Lu, 2006, 130-133). The work pressure of MSP430 SCM is very wide, and it is in $1.8 \sim 3.6 \mathrm{~V}$, but the work pressure of most SCM is $5 \mathrm{~V}$, and the range is narrow, and SCM of MSP430 offers five sort of low power consumption modes to realize the low power consumption under various conditions. For the exterior apparatus, many chips of MSP430 integrate 12bits A/D modules and Flash memory units which could reduce the complexity of exterior circuit and the power consumption of the system. The processor of MSP430F149 is one typical processor in the series, and it possesses low consumption, and its pressure is in $1.8 \sim 3.6 \mathrm{~V}$, and it could run under the condition of $1 \mathrm{MHz}$ clock, and the current consumption is in $0.1 \sim 400 \mathrm{~mA}$. The power consumption of RAM is only $0.1 \mathrm{~mA}$ under the power saving mode, and only $0.7 \mathrm{~mA}$ under the waiting mode. It adopts 16RISC structure, and its abundant addressing modes, compact kernel order, higher processing speed, large numbers of registers and data memories make it possess strong processing ability, and abundant chip interior and exterior interfaces simplify the whole circuit and reduce the power consumption and volume of sensor nodes, and it is very fit for the nodes of wireless alarming sensor.

\subsection{Module of wireless communication}

The module of wireless communication is to implement wireless communication with other nodes, exchange control information and receive collected data, and the consumed energy of wireless communication is the main part of the whole power consumption of the wireless alarming network, so the selection of this module is very important for the design of low power consumption. The tuning mode, data rate, emission power and operation cycle adopted by the wireless receiving component are key factors to influence the energy consumption of communication. And when we select the wireless receiving chip, we should consider power consumption, emission power, receiver sensibility, quantity of exterior component needed by receiving chips and chip cost. CC2420 (Wang, 2005, P. 59-62) adopted in this article is the chip which is highly integrated by low cost and low power SCM according to the standard of IEEE802.15.4, and it works in the ISM frequency band of $2.4 \mathrm{GHz}$, and it possesses $2 \mathrm{MChips} / \mathrm{s}$ direct spread spectrum basic band modulator, $250 \mathrm{~kb} / \mathrm{s}$ effective data speed, and low current consumption, and it accords with the requirement of low power supply pressure which receiving pressure is $19.7 \mathrm{~mA}$ and the transmitting pressure is $17.4 \mathrm{~mA}$. It could export power through programming and emit independent 128bits. The battery capacity of the data receiver buffer could be monitored, and the chip possesses good performance, and especially its very low current consumption could fulfill 
characters such as small volume, low power consumption and low cost of wireless alarming sensor nodes.

\subsection{Outline and consideration of sensor node design}

The part of wireless communication is the emphasis and difficulty of sensor node design and it is the key to design the alarming system. Main problems and solutions in the design process of sensor node include that the carrier waver frequency of CC2420 is $2.4 \mathrm{GHz}$, and when increasing a channel in every $5 \mathrm{MHz}$, the accuracy of crystal oscillator will influence the frequency of carrier wave and the establishment and stability of communication. CC2420 requires the precision of clock source is in $\pm 40 \times 10$. If the exterior crystal oscillator is used, we should try to use four-foot parcel crystal oscillator with high precision and stable performance. The radio-circuit of CC 2420 works in the high frequency band of $2.4000 \sim 2.4835 \mathrm{GHz}$ and the design of anti-jamming directly influences the radio-frequency and the running of all sensor nodes. When implementing wiring for the wireless communication, it is necessary to adopt reasonable wiring and multiplayer, and it is also the effective measure to enhance the ability of anti-jamming and reduce the electromagnetic jamming. The wiring needs concerning that the area that the wireless communication circuit which is not used for wiring should be filled by coppers and connected to the earth in order to offer RF screen and achieve the attention of effective anti-jamming, and the bottom of CC2420chip should connect with the earth, and many earthing poles should be used to connect with the bottom of CC2420 and the earth layer in order to reduce the delay series-wound interference and ensure the transmission of high frequency signal, and the apparatus should be distributed surround CC2420 and use small encapsulation to reduce series-wound interference and the influence of distribution parameters possibly.

\section{Design of system software}

In the intelligent home alarming system, we adopt the mechanisms of center node cycle inquiry and prominent event report. The center node will emit the parameter orders to every sensor node in certain time, and after the sensor node receives the parameter orders, they will emit data to the central node back. If emergent event happens, the sensor node could actively send report to the central node which will disposal the report and send out corresponding alarming information. The center node could set up the threshold value of sensor node to fulfill different users' requirements. In the intelligent home alarming system, every sensor node has a fixed address, and most transfer data are short messages, the formats of information include frame head, destination address, data size, data content and parity bit. The sensor node opens the power supply, initialize, establish the link and directly enter into the mode of dormancy, and when the center node receives the alarming intermitting request of sensor nodes, it will touch off the intermission, activate the sensor node to receive the bag of alarming information, then continue to enter into the dormancy after disposal, and wait to activate the sensor node again when the alarming intermitting request occurs. If there are many sensor nodes send alarming intermitting requests at the same time to the center node, and the center node will lose some requests because it is too late to treat, and after few seconds that the sensor node finds its request is not responded, it will send the request again until the center node responds. The sensor node adopts the series-communication mode. In the design of program, we mainly adopt the method of intermission to complete the receiving and sending of data, and the program flow of sensor nodes is seen in Figure 3.

The alarming information of the intelligent home alarming system easily makes mistakes in the process of transmission, and because there are many interference sources in the home, so the transmission of information is easy to be interfered, and the key to ensure the reliable running of intelligent home alarming network is the exact transmission of alarming information. In the intelligent home alarming system, two dangers will occur when nodes transfer information each other and one is how the receiver confirms whether the information is from the exact transmitter, and the other is how the transmitter confirms whether the information arrive to exact receiver, and doesn't be received by other nodes. According to the characters of wireless sensor network, we adopt the license technology to ensure the exact transmission of information (Wu, 2005, P. 753-755 \& Gong, 2004, P. 15-18). License is a data structure, and it includes the information, limitation, class, keys and other information of sensor node, and it is used to confirm the ID information, operation limitation, and necessary keys for the sensor node. There are three parts including the creation of license, the resolution and usage of license, and the memory and management of license for the usage of license. The alarming information transfer process in the intelligent home alarming system network adopting the license technology includes three approaches.

(1) The sensor node sends information with the information of the equipment to the center node.

(2) After the center node receives information, it memorizes the information and the equipment information of sensor node in a buffer area first, then compares the node information of sensor node with the interview limitation in the local memory to confirm whether the sensor node have right to send information to the center node.

(3) If the sensor node is the node which has right to send information, so the center node receives the information form the sensor node, and sends the feedback information of "exact receiving" to the sensor node. If the sensor node is the sending node without authorization, the center node will reject receiving the information and empty the buffer area. The 
license technology could ensure that the alarming information arrive the center node safely and effectively.

\section{Conclusions}

According to the characters of wireless sensor network and intelligent home, we design a sort of wireless alarming system based on wireless sensor network in this article. The wireless alarming system could supervise the safety environment of the whole home real time, and the range of the supervision includes room guard against theft, fire alarming, gas leakage, water pipe cracking and a series of unsafe factors. Once above accidents happen, the alarming system will send out corresponding alarming information, and the householder could immediately adopt effective emergency measures to treat the accident when he obtains alarming information. We establish a little alarming system to make the experiment, and the result indicates that the alarming system based on wireless sensor network possesses higher communication efficiency and better stable reliability.

\section{References}

Edgar H, Gallawy J \& Callaway E H. (2003). Wireless Sensor Network: Architectures and Protocols. New York: Auerbach Pulications.

Egan D. (2005). The Emergence of ZigBee in Building Automation and Industrial Control. Computing \& Control Engineering Journal. No.16(2). P. 14-19.

Gongwei, Wang, Lucai \& Li, Yanxiong. (2004). Intelligent Residence Alarm Technique Based on Single Chip Wireless Transceiver. Low Voltage Apparatus. No.11. P. 15-18.

Huangqiong \& Zhang, Hongke. (2006). Application Design of Wireless Sensor Network Based on IPv6. Journal of Chongqing University of Posts and Telecommunications (Natural Science). No.18(5). P. 621-624.

Lucong, Ma, Jiancang \& Wang, Jifu. (2006). The Research and Implementation of Wireless Sensor Network Node Based on ATmega128L and CC2420. Application of Electronic Technique. No.12. P. 130-133.

Sun, Liming. (2005). Wireless Sensor Network. Beijing: Tsinghua University Press. P.245.

Wang, Xiumei \& Liu, Naian. (2005). 2.4GHz RF Chip CC2420 and Its Application in ZigBee Communication. International Electronic Elements. No.3. P. 59-62.

Wu, Chengdong, Zheng, Jungang \& Liu, Daren. (2005). Study on Smart Home Network Technology Based on Wireless Sensor Network. Journal of Shenyang Jianzhu University Natural Science. No.21(6). P. 753-755.

Zhenglin, Zeng, Zhimin \& Wan, Jiping. (2005). Wireless Sensors Network Based on IEEE Standard 802.15.4. Transducer and Microsystem Technologies. No.24(7). P. 86-88.

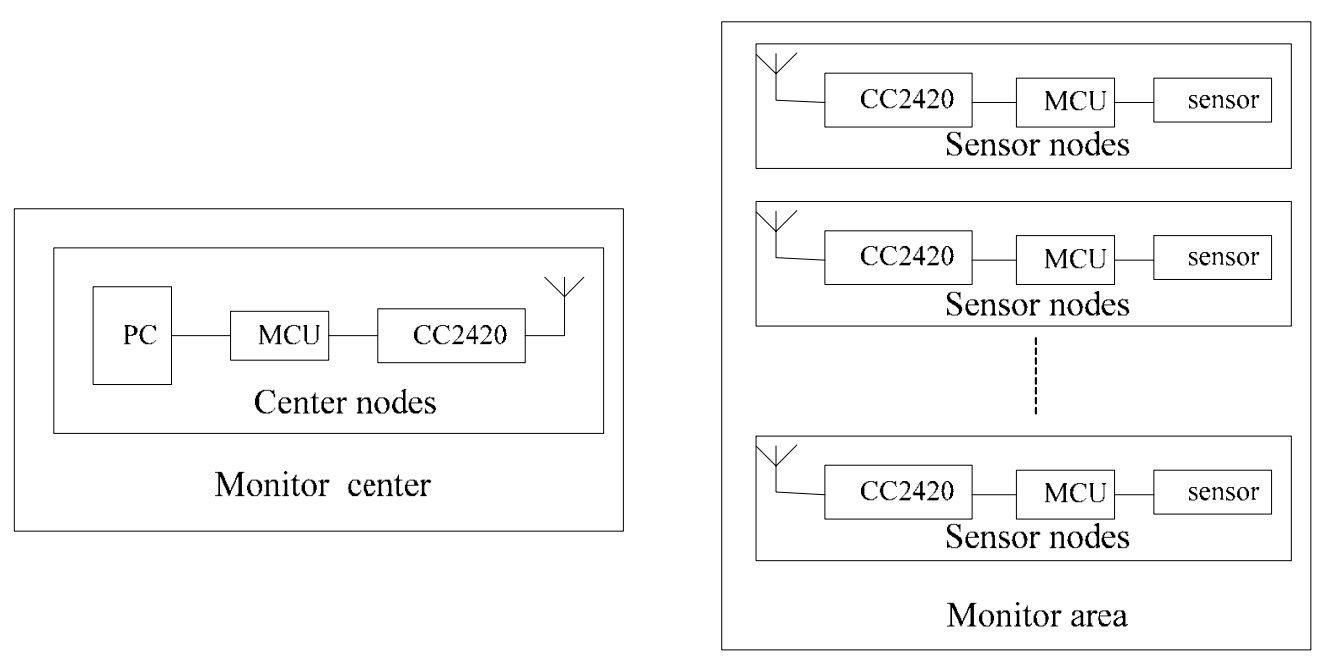

Figure 1. Intelligent Home Alarming System 


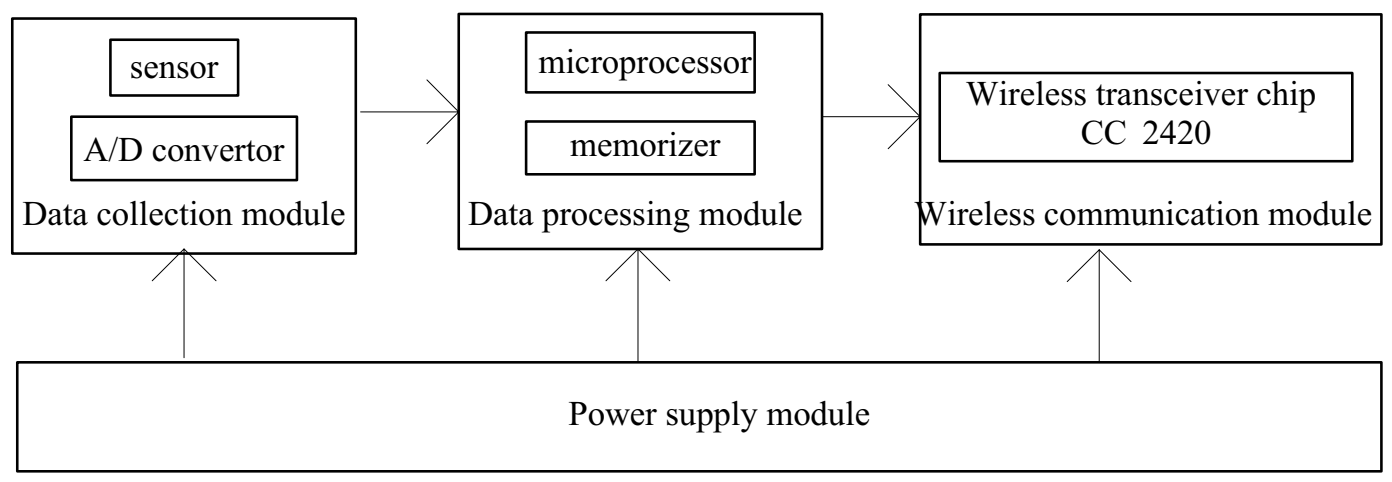

Figure 2. Structure of Sensor Nodes

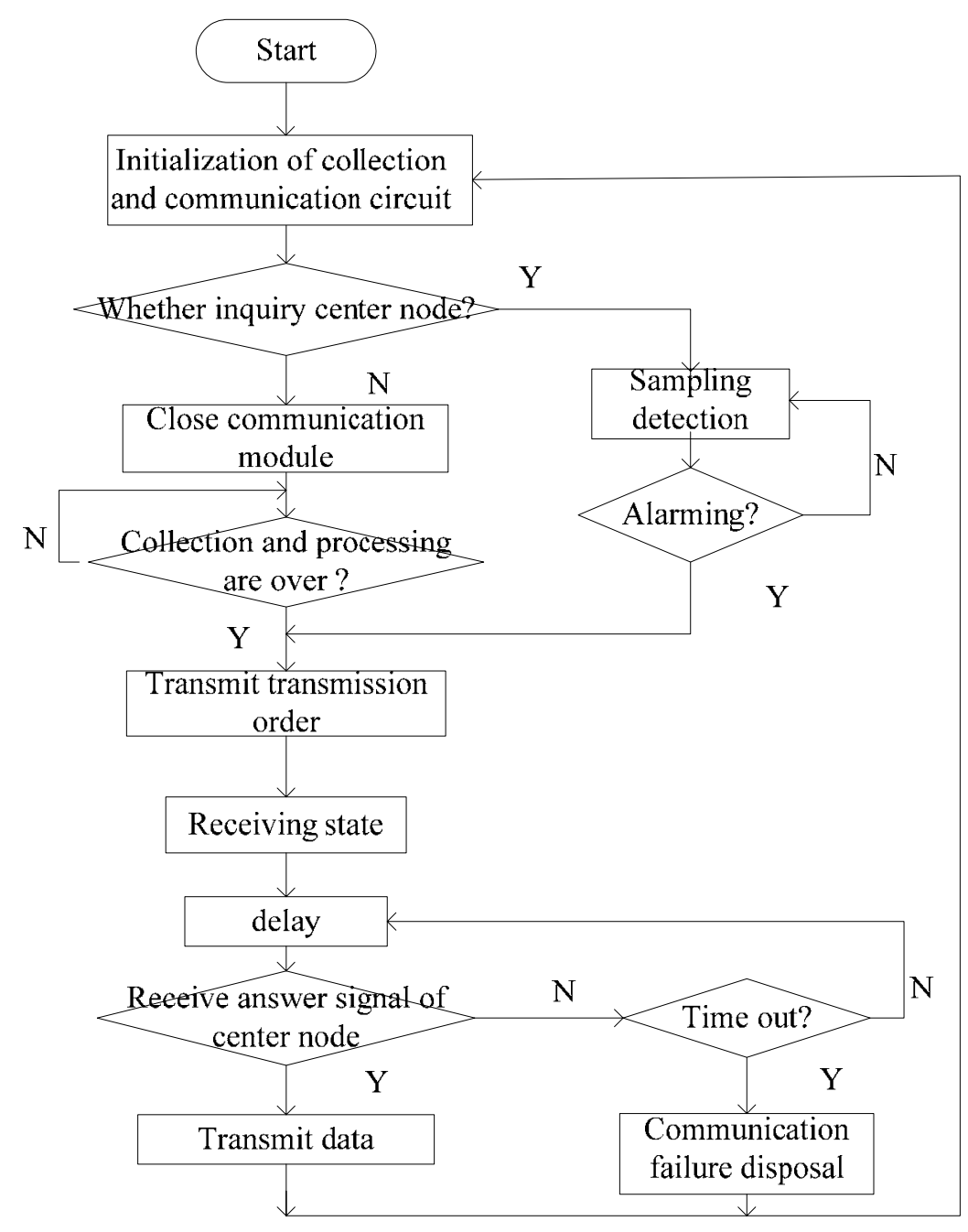

Figure 3. Flow of Sensor Nodes 